\title{
ANALYSIS OF ZONE ROUTING PROTOCOL IN MANET
}

\author{
Sandeep Kaur ${ }^{1}$, Supreet Kaur ${ }^{2}$ \\ ${ }^{I}$ M.Tech (CSE) Student, Punjabi University Regional Centre for IT \& Management Mohali, Punjab, India, \\ sandeepdeol1_5@yahoo.com \\ ${ }^{2}$ Assistant Professor (CSE), Punjabi University Regional Centre for IT \& Management Mohali, Punjab, India, \\ skaur.gujral@gmail.com
}

\begin{abstract}
MANET is combination of wireless mobile nodes that communicate with each other without any kind of centralized control or any device or established infrastructure. Therefore MANET routing is a critical task to perform in dynamic network. Without any fixed infrastructure, wireless mobile nodes dynamically establish the network. Routing Protocols helps to communicate a mobile node with the other nodes in the network by sending or receiving the packets. This research paper provides the overview of ZRP by presenting its functionality. The performance of ZRP (Zone Routing Protocol) is analyzed on the basis of parameters Throughput, Load, Data Dropped and Delay using simulator OPNET 14.0.
\end{abstract}

Index Terms: MANET, Routing Protocols, ZRP

\section{INTRODUCTION}

Computer networks were originally developed to connect number of devices through wires so that the devices can share some information and data with each other. With the increase in network sizes, the requirement of inter network communication was observed which leads to the development of internet and suit of protocols. It was necessary to provide network access to the entities which are not physically attached to any wired network. To enable this, the wireless networks were developed. Wireless network is a computer network that utilizes wireless network connection. There are two categories of wireless networks [5]:

- Infrastructured Network

- Infrastructure-less Network.

Infrastructured network contains fixed and wired gateways whereas infrastructure-less network contains multi hop wireless nodes and it has no fixed infrastructure. MANET comes under the second category. MANET [1] [2] is a temporary wireless network in which no fixed infrastructure is used. So in MANET, topology changes frequently as mobile nodes moves independently and changes their links to the other nodes very quickly. Each mobile node acts a router and forwards the traffic to the other nodes in the network. If two mobile nodes are within each other's transmission range, they can communicate directly, otherwise the nodes in between have to forward the packets for them [4].

A mobile ad hoc network may consist of only two nodes or hundred nodes or thousand nodes as well. The entire collection of nodes is interconnected in many different ways. As shown in Fig-1 there is more than one path from one node to another node. To forward a data packet from source to destination, every node in the hope must be willing to participate in the process of delivering the data packet. A single file is split it into a number of data packets and then these data packets are transmitted through the different paths. At the destination node, all these packets are combined in sequence to generate the original file. , routers

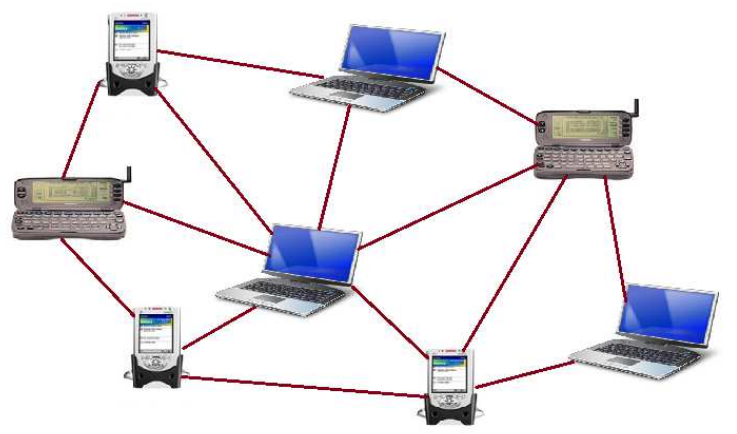

Fig-1: Mobile Ad hoc Network

\section{ROUTING IN MANET}

Routing [4] is the process of transferring a packet from source to its destination. In the routing process, a mobile node will search for a path or route to communicate with the other node in the network. Protocols are the set of rules through which two or more devices communicate with each other. In MANET, routing tables are used for routing purpose. Routing tables contain the information of routes to all the mobile nodes.

The routing protocols in MANET are broadly classified into three categories [2] [4] [6]:

- $\quad$ Proactive or Table Driven Routing Protocols

- Reactive or On-Demand Routing Protocols

- Hybrid Routing Protocols 


\subsection{Proactive or Table Driven Routing Protocols}

In Proactive or Table-Driven [8] Routing Protocols, there are routing tables which contains the information of routes to all the nodes. Routes are predefined in the routing tables and the packets are transferred to the routes. As route is already specified in the table so packet forwarding is faster and as the routes have to be defined first before transferring the packets so overhead is more. All routes are maintained at all the times so latency is low. Some highly used proactive routing protocols are Destination Sequenced Distance Vector (DSDV), Optimized Link State Routing (OLSR), Wireless Routing Protocol (WRP).

\subsection{Reactive or On-demand Routing Protocols}

In Reactive or On-Demand [1] [15] Routing Protocols, routes are not predefined. For packet transmission, a source node calls for route discovery phase to determine the route. The route discovery mechanism is based on flooding algorithm which employs on technique that a node just broadcasts the packet to all its neighbours and intermediate nodes forwards the packets to their neighbours [4]. Overhead is smaller in reactive protocols but latency is higher. Some reactive protocols are Dynamic Source Routing (DSR), Ad hoc On-Demand Distance Vector (AODV), Temporally Ordered Routing Algorithm (TORA).

\subsection{Hybrid Routing Protocols}

Hybrid Protocols [6] [7] are the combination of both i.e. Table-Driven and On-Demand protocols. These protocols take the advantage of best features of both the above mentioned protocols. These protocols exploit the hierarchical network architecture and allow the nodes with close proximity to work together to form some sort of backbone, thus increasing scalability and reducing route discovery [3]. Nodes within a particular geographical area are said to be within the routing zone of the given node. For routing within this zone, Proactive i.e. table-driven approach is used. For nodes that are located outside this zone, Reactive i.e. an on demand approach is used. So in Hybrid Routing Protocols, the route is established with proactive routes and uses reactive flooding for new mobile nodes [2]. In Hybrid Routing protocols, some of the characteristics of proactive and some of the characteristics of reactive protocols are combined, by maintaining intra-zone information proactively and inter-zone information reactively, into one to get better solution for mobile ad hoc networks [3].

\section{OVERVIEW OF ZRP}

The Zone Routing Protocol was the first Hybrid routing protocol [9] [11]. It was proposed to reduce the control overhead of Proactive routing protocol and to decrease the latency of Reactive routing protocol. It is suitable for the networks with large span and diverse mobility patterns. For each node a routing zone is defined separately. Within the routing zone, routes are available immediately but for outside the zone, ZRP employs route discovery procedure.
For each node, a separate routing zone is defined. The routing zones of neighboring nodes overlap with each other's zone. Each routing zone has a radius $\rho$ expressed in hops [9]. The zone includes the nodes whose distance from the source node is at most $\rho$ hops.

In Fig-2, routing zone of radius 2 hops for node A is shown. Routing zone includes nodes all the nodes except node L, because it lies outside the routing zone node A. The routing zone is not defined as physical distance, it is defined in hops. There are two types of nodes for a routing zone in ZRP [9]:

- Peripheral Nodes

- Interior Nodes

The nodes whose minimum distance to central node is exactly equal to the zone radius $\rho$ are Peripheral Nodes while the nodes whose minimum distance is less than the zone radius $\rho$ are Interior Nodes. In Fig. 2, Peripheral nodes are E, F, G, K, M and Interior Nodes are B, C, D, H, I, J. The node $\mathrm{L}$ is outside the routing zone of node $\mathrm{A}$.

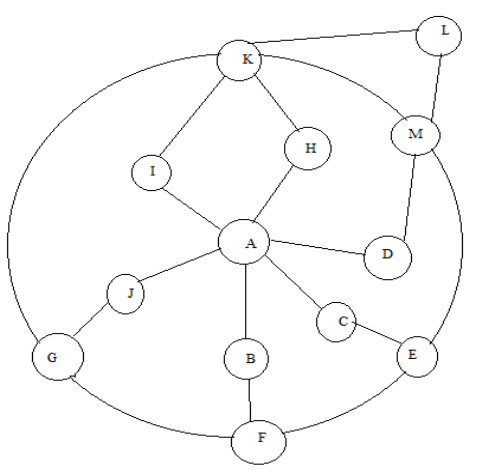

Fig-2: Routing Zone of Node A with Radius $\rho=2$ hop

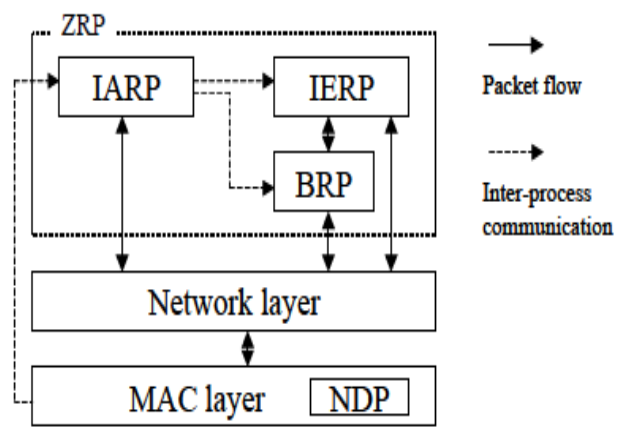

Fig-3: Architecture of ZRP [10]

The source node sends a route request to the peripheral nodes of its zone. Route request contains source address, the destination address and a unique sequence number. Each peripheral node checks its local zone for the destination. If the destination is not a member of this local zone, the peripheral node adds its own address to the route request packet and forwards the packet to its own peripheral nodes. If the destination is a member of its local zone, it sends a route reply on the reverse path back to the source. The source node uses the path saved in the route reply packet to send data packets to the destination. By adjusting the transmission power of nodes, numbers of nodes in the 
routing zone can be regulated. Lowering the power reduces the number of nodes within direct reach and vice versa [10]. ZRP uses both the strategies i.e. Proactive and Reactive routing. Within a routing zone, Proactive strategy is used. Between the routing zones, Reactive strategy is used. ZRP refers to locally proactive routing component as IntrA-zone Routing Protocol (IARP). The globally reactive routing component is named as IntEr-zone Routing Protocol [9]. Its architecture is shown in Fig-3. IARP maintains routing information of the nodes which are within the routing zone of a node. Route discovery and route maintenance is offered by IERP. When global discovery is needed, if the topology of local zone is known, it can be used to reduce the traffic. Instead of broadcasting a packet, ZRP uses the concept of Bordercasting [10]. Bordercasting packet service delivery is provided by the Bordercasting Resolution Protocol (BRP). The BRP [11] uses a map of an extended routing zone, provided by the local IARP, to construct Bordercast trees along which query packets are directed. The BRP uses very special query control mechanisms to steer route request away from areas of the network that have already covered by the query [11].

\subsection{Route Discovery Process}

The discover process of ZRP operates as follows [10] [12]:

- The source node first checks whether the destination is within its zone. If so, destination node is known and no further route discovery process is required.

- If the destination is not within the routing zone of source, the source node bordercast a route request to its peripheral nodes.

- The peripheral nodes checks whether the destination node is within their node or not. If so, a route reply is sent back to the source node indicating the route to the destination.

- If the destination node is not available in the routing zones of peripheral nodes, route requests are forwarded to their peripheral nodes.

The route discovery process is shown in the Fig-4.

\subsection{Route Maintenance}

Route maintenance is important in ad hoc networks, in which links are broken and established as nodes moves relatively to each other with limited radio coverage. Route discovery or route repair must be performed if the route broken or fails. Until the new route is available, packets are dropped or delayed. [9]

In ZRP, the knowledge of the local topology can be used for route maintenance. Link failures and sub-optimal route segments within one zone can be bypassed. Incoming packets can be directed around the broken link through an active multi-hop path. The topology can be used to shorten the routes, for example, when two nodes have moved within each other's radio coverage. For routed packets, a relaying node can determine the closet route to the destination that is also a neighbour. [9]

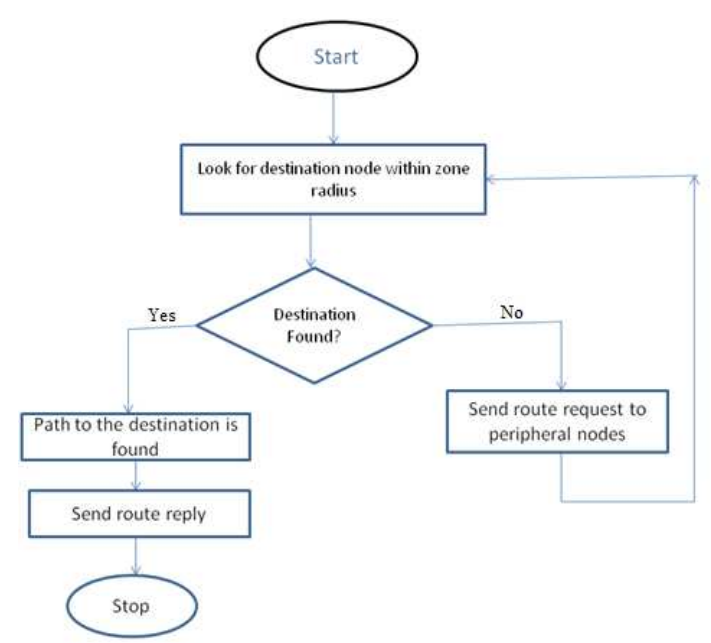

Fig-4: Flowchart of Route Discovery Process

\section{SIMULATION SETUP}

\subsection{Simulation Scenario}

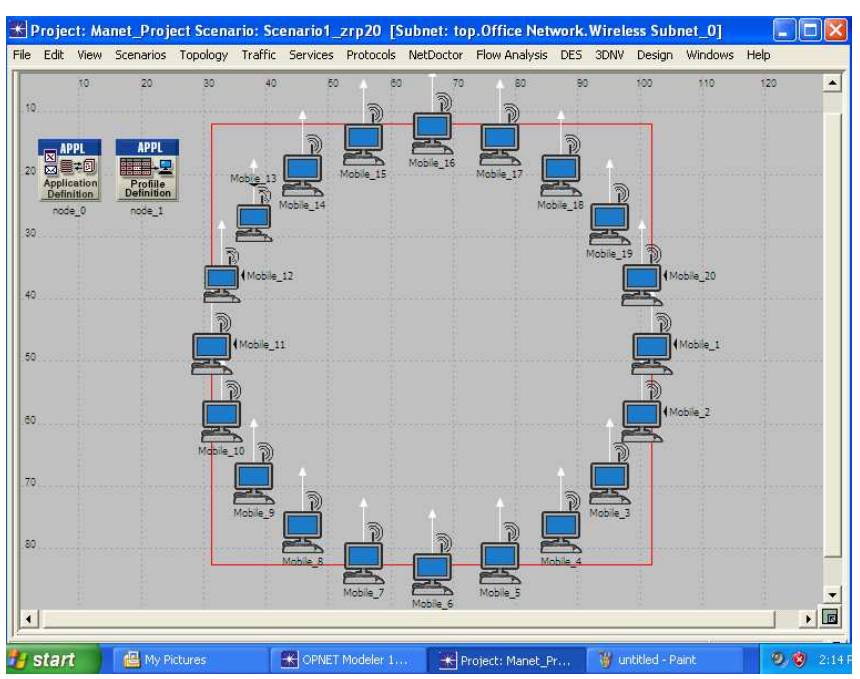

Fig-5: Simulation Scenario with 20 nodes

To analyse the performance of ZRP OPNET 14.0 simulator is used. Three different scenarios are created with varying number of mobile nodes. The three scenarios contain 20, 40 and 60 mobile nodes respectively. The pause time and traffic load are kept constant under all the scenarios. Simulation parameters used for the implementation of ZRP are listed in the Table 1.

\subsection{Performance Metrics}

Throughput [2]: Throughput is the average rate of successful data packets received at the destination [2]. It is the measure of how fast we can actually send the packets through the 
network. It is measured in bits per second (bits/sec or bps) or data packets per second.

Load [6]: Load in the wireless LAN is the number of packets sent to the network greater than the capacity of the network. When the load is less than the capacity of the network, the delay in packets is minimum. The delay increases when the load reaches the network capacity.

Data Dropped: Data dropped is the count of number of bits per second which are dropped during the travelling of signals from source to destination. Data can be dropped due to unavailability of access to medium.

Delay [7]: The packet end-to-end delay refers to the time taken for a packet to be transmitted across the network from source to destination. In other words, it is the time a data packet is received by the destination minus the time a data packet is generated by the source. It is measured in seconds. End. Lost packets due to delay have a negative effect on received quality.

Table-1: Simulation Parameters

\begin{tabular}{|c|c|}
\hline Parameters & Values \\
\hline Simulator & OPNET 14.0 \\
Examined & ZRP \\
\hline Simulation Time & 300 seconds \\
\hline Simulation Area & $1000 \mathrm{~m} \times 1000 \mathrm{~m}$ \\
\hline Pause Time & 10 sec \\
\hline Buffer Size (bits) & 256000 \\
\hline Data Rate (bps) & $11 \mathrm{Mbps}$ \\
\hline Mobility Model & Random way \\
& point \\
\hline Mobile Nodes & $20,40,60$ \\
\hline
\end{tabular}

\section{RESULTS \& DISCUSSION}

Throughput: It is observed from the Fig- 6 that with 20 nodes the throughput of ZRP is about 20000 bits per second till 1 minute and 40 seconds after that its value increases to $1,20,000$ bits per second. With 40 and 60 nodes it gives zero throughput till 1 minute and 40 second. After that the throughput of ZRP is gradually increasing and fluctuating. Maximum value of throughput of $10,000,000$ is observed at 3 minute with 60 nodes.

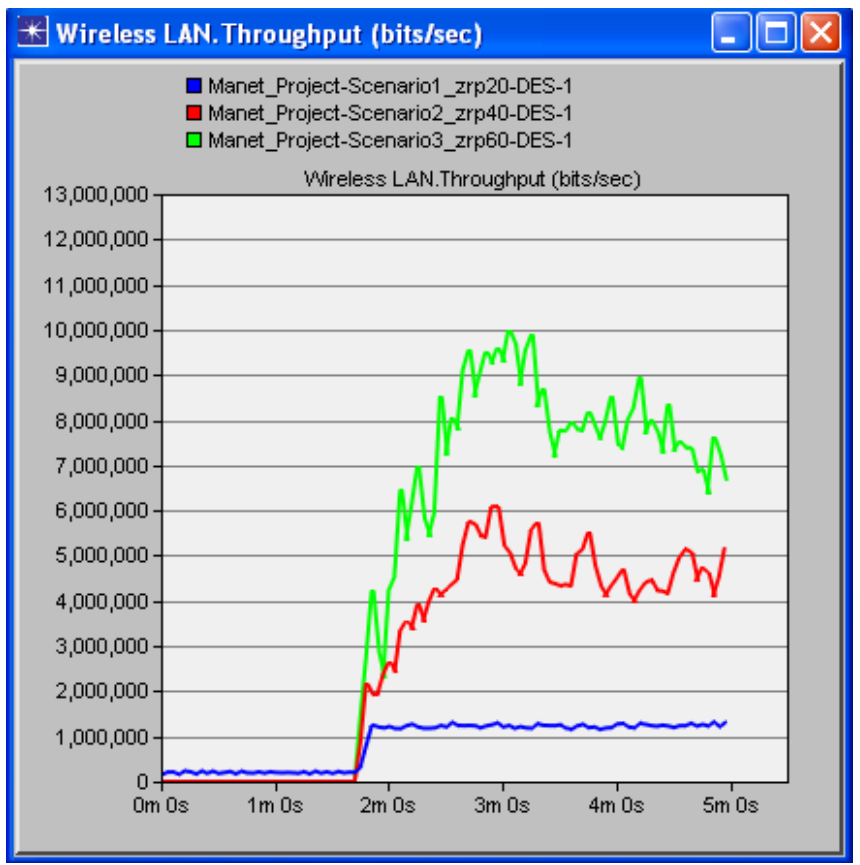

Fig-6: Throughput of ZRP

Load: From Fig-7 it is observed that load of ZRP is 0 bits per second till 1 minute and 40 seconds for 20, 40 and 60 nodes. Minimum load is observed with 20 nodes. With 40 and 60 nodes, a high load is observed. Load with 40 and 60 nodes is almost the same. Maximum load of 66,00,000 bits per second is observed with 60 nodes at 4 minute.

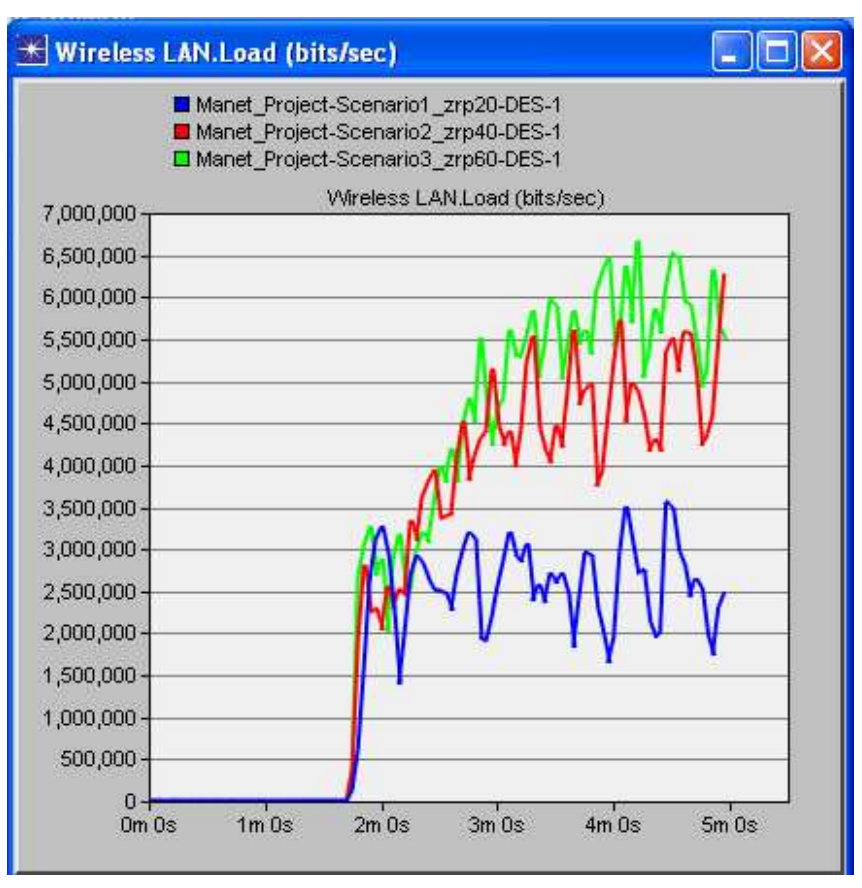

Fig-7: Load of ZRP

Data Dropped: It is observed from the Fig- 8 no data is dropped till 1 minute and 40 seconds. Afterwards data is dropped till the end of the simulation. Low data is dropped with 20 nodes. With 40 and 60 nodes the data dropped rate is almost same. Its value is fluctuates between 3,000,000 
bits per second to $58,000,000$ bits per second for 40 and 60 nodes. Maximum data dropped rate about 57,000,000 bits per second is observed at 4 minute.

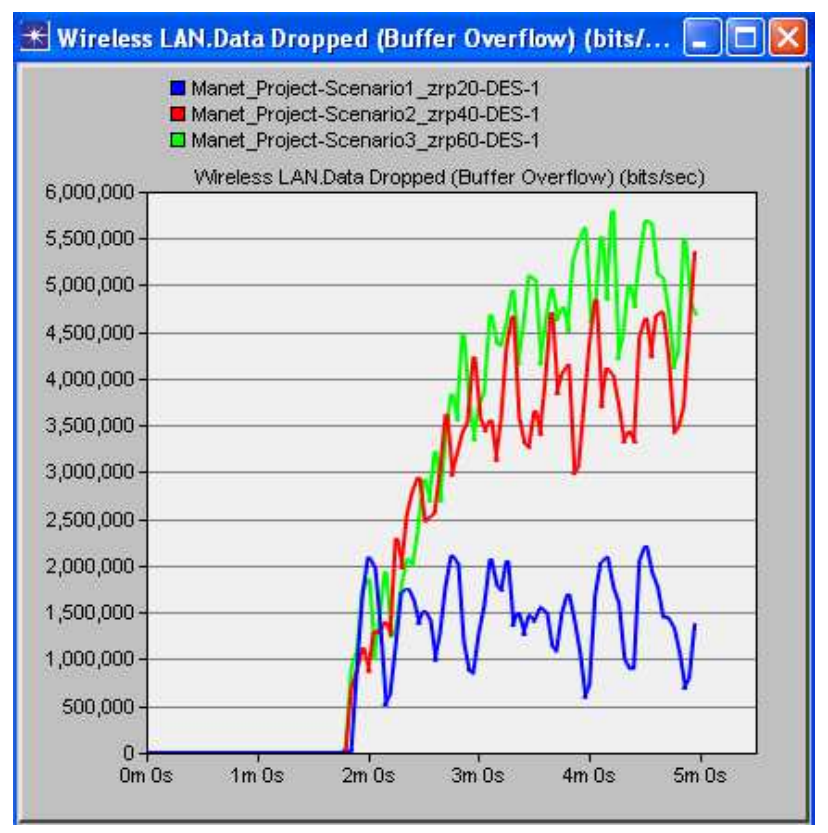

Fig-8: Data Dropped

Delay: From Fig-9, it is observed that delay of ZRP is high with 60 nodes. Average delay is observed with 40 nodes and minimum delay is observed with 20 nodes. High delay of 19 sec is observed with 60 nodes.

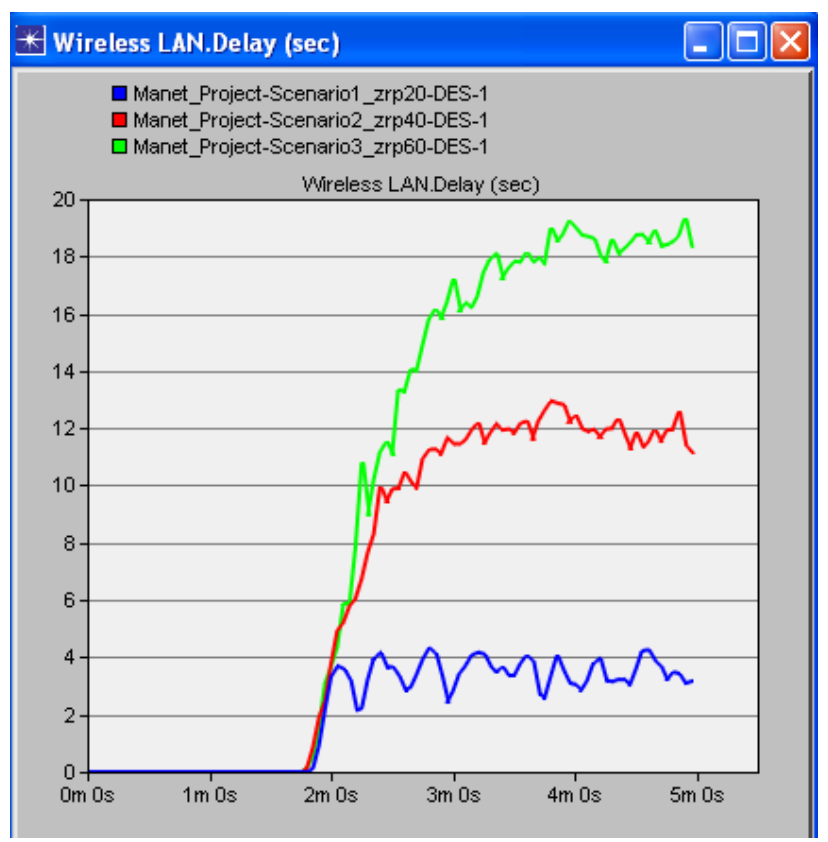

\section{CONCLUSIONS}

Fig-9: Delay

From the results it is concluded that with the increase in number of mobile nodes, ZRP gives high throughput. Load increases with the increase in nodes. With 20 nodes, it gives minimum load but as the nodes increases, a high load is observed. With high load, the delay is also high. Data dropped also increases with the increase in number of nodes.

\section{REFERENCES}

[1] Nadia Qasim, Fatin Said, Hamid Aghvami, "Mobile Ad Hoc Networks Simulations Using Routing Protocols for Performance Comparisons", Proc. of the World Congress on Engineering, vol. 1, WCE 2008, July 2008.

[2] Kuncha Sahadevaiah, Oruganti Bala, Venkata Ramanaiah, "An Empirical Examination of Routing Protocols in Mobile Ad Hoc Networks", Proc. of International Journal of Communications, Network and System Sciences, June 2010.

[3] Humayun Bakht, "Survey of Routing Protocols for Mobile Ad-hoc Network", Proc. of International Journal of Information and Communication Technology Research, vol. 1, no. 6, October 2011.

[4] Hongbo Zhou, "A survey on Routing Protocols in MANETs", Proc. of Michigan State University, MSUCSE-03-08, March 2003.

[5] Geetha Jayakumar† and Gopinath Ganapathy, "Performance Comparison of Mobile Ad-hoc Network Routing Protocol", Proc. of International Journal of Computer Science and Network Security, vol. 7, no. 11, Novemember 2007.

[6] Kavita Panday, Abishek Swaroop, "A Comprehensive Performance Analysis of Proactive, Reactive and Hybrid MANETs Routing Protocols", Proc. of International Journal of Computer Sciences Issues, vol. 8, Issue 6, no. 3, Nov 2011.

[7] Parma Nand, Dr. S.C. Sharma, "Comparative study and Performance Analysis of FSR, ZRP and AODV Routing Protocols for MANET”, Proc. of International Journal of Computer Applications, 2011.

[8] Ashish K. Maurya, Dinesh Singh, "Simulation based Performance Comparison of AODV, FSR and ZRP Routing Protocols in MANET', Proc. of Internationl Journal of Computer Applications, vol.12, no.2, November 2010.

[9] Zygmunt J. Haas, "The Zone Routing Protocol (ZRP) for Ad hoc Networks", Internet Draft, July 2002.

[10] Nicklas Beijar, (2002) "Zone Routing Protocol (ZRP)" [Online].

Available: http://www.netlab.tkk.fi/opetus/s38030/k02/Papers/08Nicklas.pdf

[11] Sanku Sinha, Biswaraj sen, "Effect of Varying Node Density and Routing Zone Radius in ZRP: A Simulation Based Approach", Proc. of International Journal on Computer Science and Engineering (IJCSE), vol.4, no.6, June 2012.

[12] M.N. Sree Ranga Raju, Dr. Jitendranath Mungara, "Enhanced ZRP Protocol for Mobile Ah hoc Networks", Proc. of International Journal of Wireless and Mobile Networks, vol.3, no.4, August 2011. 